\title{
Pathways related to PMA-differentiated THP1 human monocytic leukemia cells revealed by RNA-Seq
}

\author{
ZENG ChengWu ${ }^{1,3,4 \dagger}$, WANG WenTao ${ }^{2 \dagger}, \mathrm{YU} \mathrm{XiBao}^{4}, \mathrm{YANG}_{\mathrm{LiJian}}{ }^{3}, \mathrm{CHEN} \mathrm{ShaoHua}{ }^{3}$ \\ \& LI YangQiu ${ }^{1,3,4 *}$ \\ ${ }^{1}$ First Affiliated Hospital, Jinan University, Guangzhou 510632, China; \\ ${ }^{2}$ Key Laboratory of Gene Engineering of the Ministry of Education, State Key Laboratory for Biocontrol, School of Life Science, Sun Yat-sen \\ University, Guangzhou 510275, China; \\ ${ }^{3}$ Institute of Hematology, Medical College, Jinan University, Guangzhou 510632, China; \\ ${ }^{4}$ Key Laboratory for Regenerative Medicine of Ministry of Education, Jinan University, Guangzhou 510632, China
}

Received August 6, 2015; accepted August 19, 2015; published online November 17, 2015

\begin{abstract}
Previous analyses have reported that the human monocytic cell line THP1 can be differentiated into cells with macrophage-like characteristics by phorbol 12-myristate 13-acetate (PMA). However, little is known about the mechanism responsible for regulating this differentiation process. Here, we performed high-throughput RNA-Seq analysis to investigate the genes differently expressed in THP1 cells treated with and without PMA and examined those that may be responsible for the PMA-induced differentiation of monocytes into macrophages. We found 3,000 genes to be differentially expressed after PMA treatment. Gene ontology analysis revealed that genes related to cellular processes and regulation of biological processes were significantly enriched. KEGG analysis also demonstrated that the differentially expressed genes (DEGs) were significantly enriched in the PI3K/AKT signaling pathway and phagosome pathway. Importantly, we reveal an important role of the PI3K/AKT pathway in PMA-induced THP1 cell differentiation. The identified DEGs and pathways may facilitate further study of the detailed molecular mechanisms of THP1 differentiation. Thus, our results provide numerous potential therapeutic targets for modulation of the differentiation of this disease.
\end{abstract}

acute myeloid leukemia, differentiation, macrophage, PI3K/AKT pathway, RNA sequencing

Citation: Zeng CW, Wang WT, Yu XB, Yang LJ, Chen SH, Li YQ. Pathways related to PMA-differentiated THP1 human monocytic leukemia cells revealed by RNA-Seq. Sci China Life Sci, 2015, 58: 1282-1287, doi: 10.1007/s11427-015-4967-4

Acute myeloid leukemia (AML) is a hematologic disease characterized by abnormal proliferation, apoptosis repression and differentiation blockage in hematopoietic stem/progenitor cells [1]. One approach for the treatment of leukemia is chemical-induced differentiation. Because of the relationship between the differentiation of leukemia blasts and disease regression, this treatment is clinically referred to as differentiation therapy [2]. Retinoic acid (RA)

$\dagger$ Contributed equally to this work

*Corresponding author (email: yangqiuli@hotmail.com) treatment for acute promyelocytic leukemia has been considered a clinical success for differentiation therapy. Although several transcription factors have been identified to play important roles in leukemogenesis [3,4], the aberrant expression of oncogenes and disruption of tumor suppressor genes are most likely involved in processes responsible for blocking leukemia cell differentiation [5-11].

Treatment of human monocytic leukemia cells with PMA can overcome blocks in terminal differentiation, such as that in myeloid lineage THP1 cells, and result in growth arrest and differentiation into an adherent monocytic phenotype. 
Understanding the dynamics of the transcriptome during differentiation will allow us to identify novel therapeutic targets. Transcriptional profiling has proven to be useful for determining general patterns of differential gene expression among samples [12]. Recent reports have shown that RNA-Seq is highly sensitive and can quantitatively measure gene expression in a large dynamic range of gene abundance [5,13-15].

The aim of this study was to gain an understanding of the molecular mechanisms associated with the PMA-induced differentiation of monocytes into macrophages and establish a sound foundation for future molecular studies.

\section{Materials and methods}

\subsection{Cell culture and reagents}

The human monocytic leukemia cell line THP1 was cultured in RPMI 1640 (HyClone, SH30027, USA) containing $10 \%$ fetal bovine serum (HyClone, SV30160). The cells were maintained in a humidified atmosphere containing $5 \%$ $\mathrm{CO}_{2}$ at $37^{\circ} \mathrm{C}$. PMA was purchased from Sigma (USA) and dissolved in dimethyl sulfoxide (DMSO). Cell differentiation was assessed by measuring surface marker integrin alpha M (ITGAM/CD11b) and CD14 molecule (CD14) expression (eBioscience, 11-0113, USA) by flow cytometry analysis.

\subsection{Quantitative real-time PCR analysis}

RNA was reverse-transcribed into cDNA using M-MLV reverse transcriptase (Promega, M1705, USA). PCR of the cDNA was performed using specific primers for the genes of interest. The oligonucleotide sequences are available in Table S1. GAPDH (glyceraldehyde-3-phosphate dehydrogenase) was used as an internal control.

\subsection{Cell counting kit-8 (CCK-8) assay}

THP1 cells were plated at a density of $5 \times 10^{3}$ cells/well in 96 well plates, and cell viability was assessed using the CCK-8 assay according to the manufacturer's instructions. After treatment with LY294002 (PI3K/Akt inhibitor) and PMA for 24, 48, and $72 \mathrm{~h}$, the CCK-8 (Dojingdo, Japan) reagent $(10 \mu \mathrm{L})$ was added to each well followed by a 2-hour incubation.

\subsection{RNA isolation and RNA-Seq (quantification)}

To obtain an overview of the macrophage differentiation transcriptome, cDNA libraries generated from THP1 cells stimulated with PMA at several time points were used. The cDNA libraries were prepared as previously described [16]. The library products were used for sequencing by the Illu- mina HiSeq ${ }^{\mathrm{TM}} 2000$ platform (USA). Gene expression levels were calculated by assessing the number of uniquely mapped reads per kilobase of exon region per million mappable reads (RPKM) using Chen's method [17]. RPKM values were used to compare differences in gene expression among samples. We identified differentially expressed genes (DEGs) between samples using the following criteria: FDR (false discovery rate) $\leqslant 0.001$ and $\log _{2}$ fold-change ratio $\geqslant 1$.

\section{Results}

\subsection{Differentially expressed genes in monocytes and macrophages}

Treatment of THP1 cells with PMA is a well-established model for monocyte/macrophage differentiation. THP1 cells were treated with PMA $\left(50 \mathrm{ng} \mathrm{mL}^{-1}\right)$ for 24,48 and $72 \mathrm{~h}$. A marked increase in cell size and adhesion was observed for PMA-treated THP1 cells, which had a typical macrophage shape and signs of macrophage differentiation (Figure S1). Next, we performed RNA sequencing (RNA-Seq) to identify the genes involved in macrophage differentiation. The gene expression profiles derived from RNA-Seq were calculated using the RPKM method. There were approximately 20,000 genes detected on average in the individual libraries, and greater than 3,000 genes were differentially expressed after PMA treatment. Using hierarchical clustering analysis, we found that a large number of genes were robustly induced during the process of macrophage differentiation (Figure 1), including $p 21^{\text {WAFl/Cipl }}$, MCL1 (myeloid cell leukemia 1) and BCL2L1 (BCL2-like 1), which were previously reported to be induced during differentiation [18-20]. The DEGs are shown in Table $\mathrm{S} 2$.

\section{2 qRT-PCR for data validation}

To validate the DEGs identified by RNA-Seq, we assessed nine of the DEGs (i.e., ITGAM/CD11b, CD14, SPIB (Spi-B transcription factor), $V E G F$ (vascular endothelial growth factor), MMP9 (matrix metallopeptidase 9), HOXA9 (homeobox A9), ATG7 (autophagy related 7), CXCR4 (chemokine (C-X-C motif) receptor 4), and EGRl (early growth response 1)) that exhibited highly significant differences in expression or were involved in important signaling pathways by quantitative RT-PCR. The results of the qRT-PCR analysis were generally in good agreement with the RNA-Seq data (Figure 2). Importantly, RT-PCR detection demonstrated that the mRNA level of some genes, including the macrophage lineage surface markers ITGAM/CD11b and CD14, increased in response to PMA, which is consistent with previous studies $[1,18]$. These results confirmed that RNA-Seq could provide reliable data for differential RNA expression analysis. 


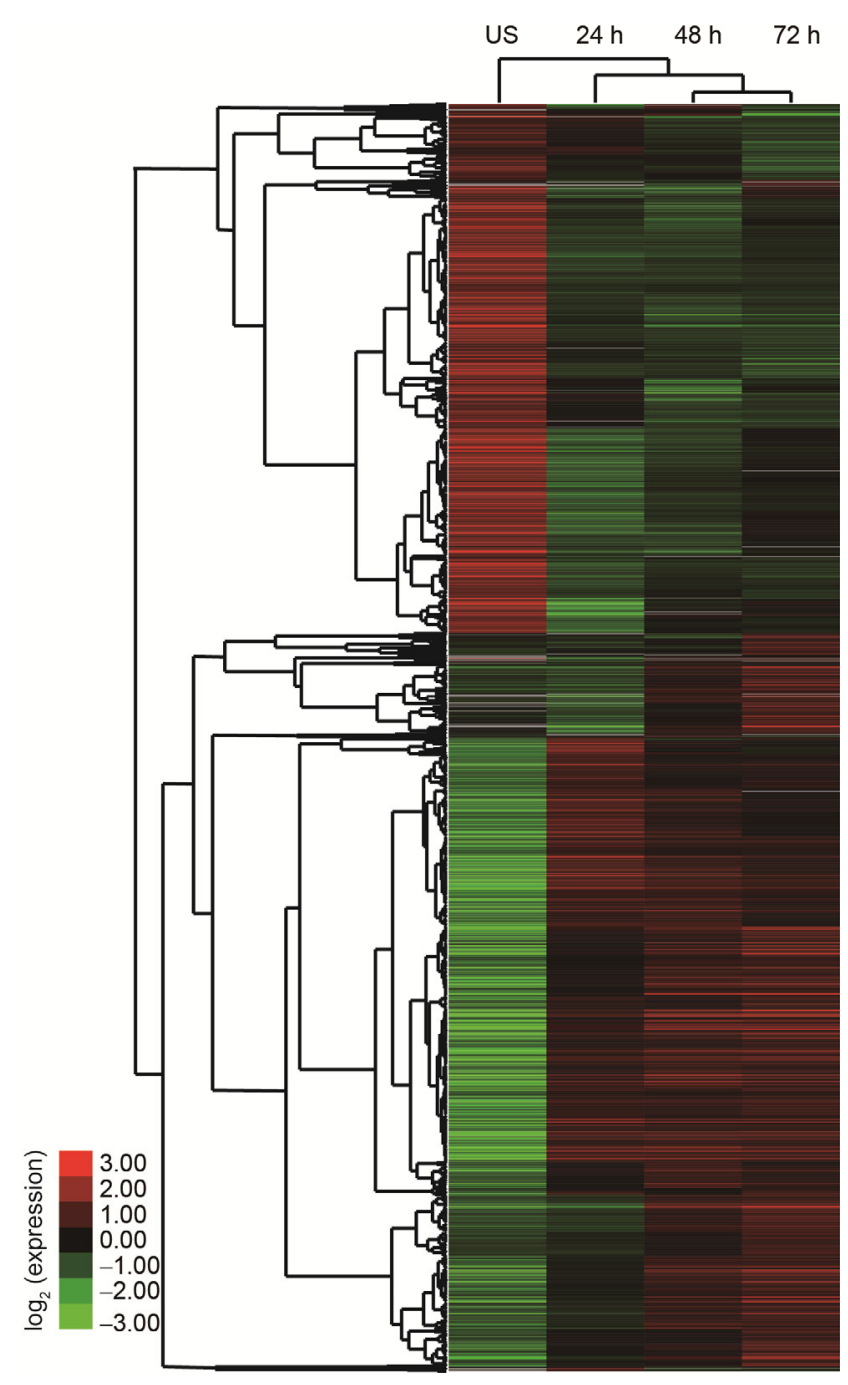

Figure 1 Hierarchical clustering analysis of differentially expressed genes. The heatmap shows hierarchical clustering of the genes expressed during the differentiation of monocytes into macrophages. The color intensity represents the degree of expression. A red-green color scale was used to reflect standardized gene expression with red representing high expression and green representing low expression (scale shown in the lower left). Cutoff values of $\mathrm{FDR} \leqslant 0.001$ and $\log _{2}$ fold-change ratio $\geqslant 1$ relative to unstimulated cells were selected. The identities of these genes are listed in Supplemental table 2. US: unstimulated.

\subsection{Gene ontology (GO) and KEGG pathway enrich- ment analysis of DEGs}

We performed GO and KEGG analysis to classify the functions of the DEGs. To avoid experimental variation, we only used DEGs that had a $\log _{2}$ fold-change ratio $\geqslant 2$. We compared the three time points of the PMA-treated and untreated cells. Analysis of the DEGs based on the biological process GO categories revealed that genes related to cellular processes and the regulation of biological processes were significantly enriched. The most significant biological process terms are listed in Table S3.

Pathway-based analysis helps to further understand the biological functions of DEGs. The KeggChart tool in the DAVID program was used to detect enriched pathways in the KEGG database [21]. Several pathways were found to be significantly enriched for in the DEGs (Table S4). Pathways most commonly reported to be related to proliferation and differentiation, such as the PI3K/AKT, Notch, MAPK and NF- $\mathrm{B}$ signaling pathway, were identified [19,22-24]. Importantly, the PI3K/AKT signaling pathway was found to be significantly enriched for in the DEGs. These findings are particularly interesting because the PI3K/AKT signaling pathway has been closely linked with PMA-induced $\mathrm{p} 21^{\mathrm{WAF} 1 / \mathrm{Cip} 1}$ expression in human leukemia cells [20].

\subsection{The PI3K/AKT pathway in PMA-induced THP1 cell differentiation and growth inhibition}

Studies of human leukemia cell lines have indicated that PMA inhibits THP1 cell proliferation before the initiation of differentiation occurs [25]. Interestingly, previous reports have also shown that the PI3K/AKT pathway plays an important role in regulation of $\mathrm{p}^{21 \mathrm{WAF} 1 / \mathrm{Cip} 1}$ expression during prostate cancer cell proliferation [26]. We therefore asked whether PMA could induce differentiation via PI3K/AKT pathway-dependent proliferation inhibition. We examined the effects of LY294002 (PI3K/AKT inhibitor) on cell proliferation and differentiation in PMA-stimulated THP1 cells by measuring CD11b and CD14 surface antigen expression. As shown in Figure 3A, we found that LY294002 treatment inhibits PMA-induced THP1 cell differentiation but has no effect on THP1 cell growth (Figure 3B). Collectively, these results further support the data of our RNA-Seq analysis and suggest that the effects of the PI3K/AKT pathway on THP1 cell differentiation may not depend on growth inhibition.

\section{Discussion}

In this study, we examined the molecular mechanisms associated with the PMA-induced differentiation of monocytes into macrophages. For this purpose, THP1 macrophages generated by stimulation with PMA at several time points were used. We obtained the comprehensive transcriptome of the process of monocyte-to-macrophage differentiation using RNA-Seq. Additionally, genes differentially expressed during the different stages of the PMA-induced differentiation of monocytes into macrophages were identified and functionally annotated using the GO and KEGG databases.

To elucidate the fundamental roles of these DEGs, we analyzed putative genes using GO "biological process" categories. This analysis revealed that a set of DEGs related to cellular processes and regulation of biological processes may be associated with macrophage function. The enriched KEGG pathways associated with the DEGs clustered into 

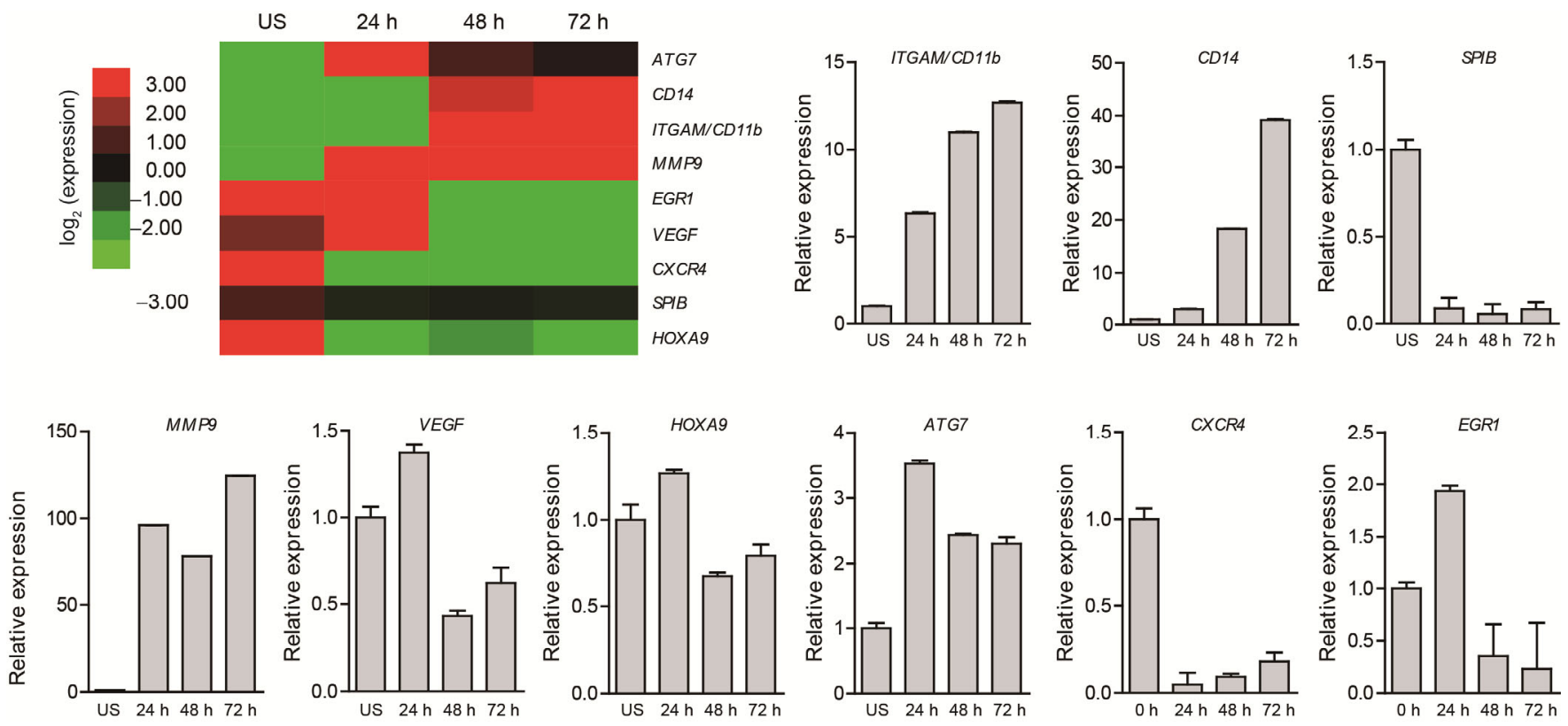

Figure 2 Quantitative real-time PCR validation of RNA-Seq data. The color panels indicate the $\log _{2}$-transformed mean expression values obtained by RNA-Seq (see reference color bar). mRNA levels were measured by qRT-PCR and are shown as n-fold changes compared with untreated cells normalized to the housekeeping gene $G A P D H$. The panels show the $\bar{x} \pm \mathrm{SD}$ of a representative experiment performed in triplicate.
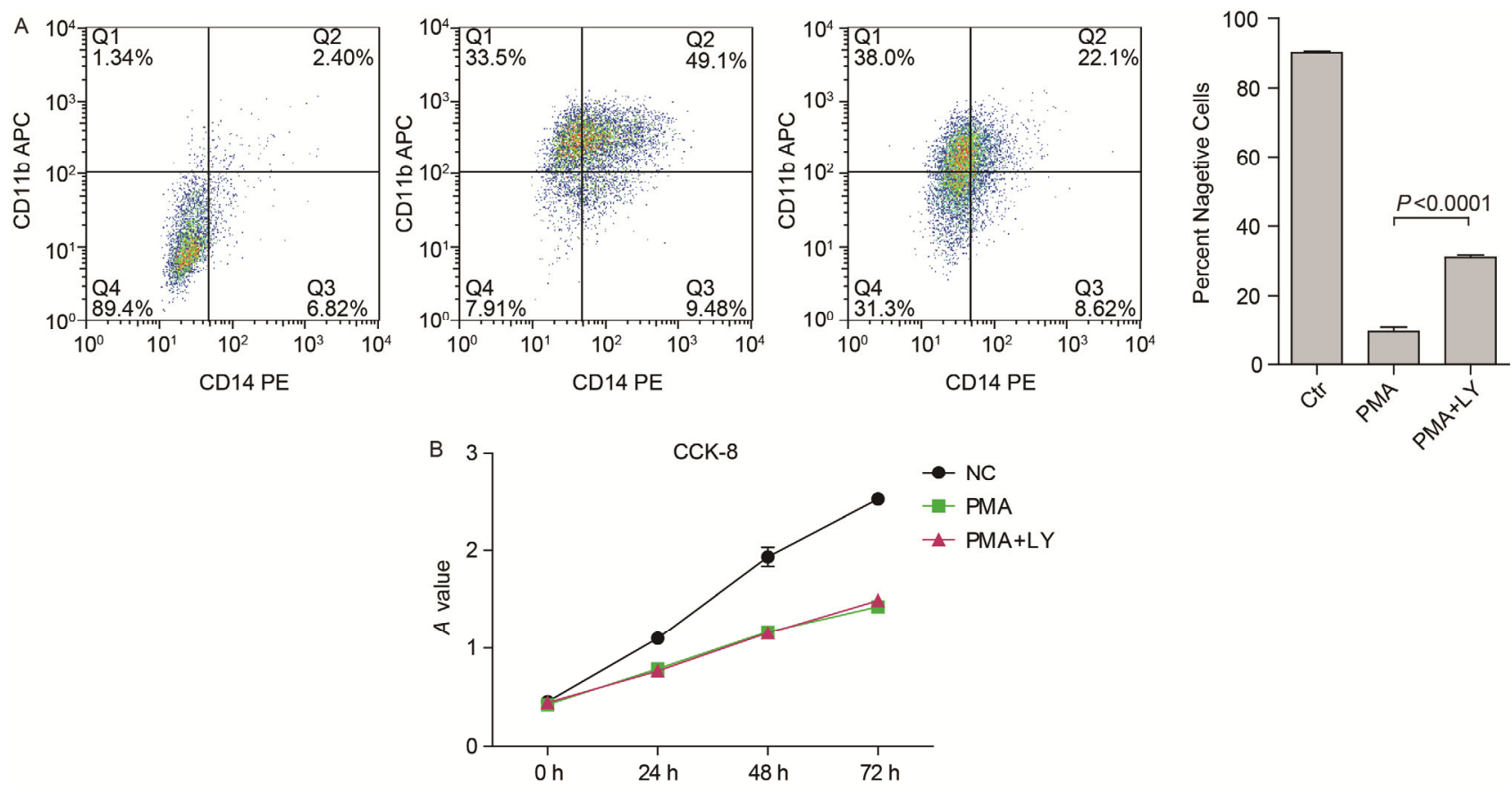

Figure 3 Effects of LY294002 on PMA-induced THP1 cell differentiation and growth. A, THP1 cells were seeded in 6-well plates in RPMI containing $10 \%$ fetal bovine serum, and they were treated with PMA $\left(50 \mathrm{ng} \mathrm{mL}^{-1}\right)$ alone or in combination with LY294002 $\left(10 \mu \mathrm{mol} \mathrm{L}^{-1}\right)$ for $48 \mathrm{~h}$. Following differentiation, the cells were evaluated for cell-surface markers (CD11b and CD14) by flow cytometry. The values shown are derived from $n=3$ independent experiments, and data are reported as $\bar{x}_{ \pm \mathrm{SD}}$. B, THP1 cells were co-treated with LY294002 and PMA $\left(50 \mathrm{ng} \mathrm{mL}^{-1}\right)$ or PMA alone for the indicated times. Cell viability was analyzed using the CCK- 8 assay.

several categories. We demonstrated that the most widely reported pathways related to proliferation and differentiation, such as the PI3K/AKT and NF- $\kappa \mathrm{B}$ signaling pathways, were also included. Of these pathways, we were particularly interested in the PI3K/AKT pathway, which was significantly activated. A recent study has reported that PI3K/ AKT regulates survival during the differentiation of human macrophages [19]. Interestingly, by measuring CD11b and 
CD14 surface antigen expression, we found that inhibition of PI3K activity by LY294002 inhibits PMA-induced THP1 cell differentiation. Inhibition of the PI3K/ AKT pathway suppressed the PMA-induced morphological changes, suggesting that the PI3K/AKT pathway is required for PMA-induced THP1 differentiation. It is understood that before initiating cell differentiation, PMA treatment must first inhibit cell growth [25]. Regulation of $\mathrm{p} 21^{\mathrm{WAF} 1 / \mathrm{CIP} 1}$ gene expression has been extensively studied in macrophage differentiation [20]. $p 21^{\text {WAFI/CIPI }}$ gene expression was upregulated in our RNA-Seq dataset, which is similar to other reports. Previous reports have shown that the PI3K/AKT pathway plays an important role in regulation of p21 $1^{\text {WAFI/Cipl }}$ expression during proliferation [26]. We therefore tested the effects of LY294002 on cell proliferation during PMA-stimulated differentiation. However, we found that LY294002 and PMA co-treatment had no effect on THP1 cell growth during macrophage differentiation. Previously reported results in differentiated macrophages demonstrated that a signaling pathway consisting of $\mathrm{PI} 3 \mathrm{~K} / \mathrm{Akt}$ and NF- $\kappa \mathrm{B}$ regulates cell survival during the differentiation process [19]. Indeed, we found significant changes in BCL2 family genes in our data (Table S5). Thus, the functions of the PI3K/AKT pathway in the differentiation of PMA-treated THP1 cells may not depend on growth inhibition.

In conclusion, in this study, by using RNA-Seq to evaluate the effects of PMA at different time points, we identified a number of genes associated with the PMA-induced differentiation of monocytes into macrophages. Functional and pathway analyses of the genes further demonstrated the importance of the PI3K/AKT signaling pathway in regulating the differentiation process. These data will provide potential molecular targets for functional studies.

The author(s) declare that they have no conflict of interest.

This work was supported by funds from the National Natural Science Foundation of China (81400102), the Chinese Postdoctoral Science Foundation (2015M570751), the National Undergraduate Training Program for Innovation and Entrepreneurship (201510559043), and the Medical Scientific Research Foundation of Guangdong Province, China (A2015420).

1 Pession A, Martino V, Tonelli R, Beltramini C, Locatelli F, Biserni G, Franzoni M, Freccero F, Montemurro L, Pattacini L, Paolucci G. $M L L-A F 9$ oncogene expression affects cell growth but not terminal differentiation and is downregulated during monocyte-macrophage maturation in AML-M5 THP-1 cells. Oncogene, 2003, 22: 8671-8676

2 Ablain J, de The H. Revisiting the differentiation paradigm in acute promyelocytic leukemia. Blood, 2011, 117: 5795-5802

3 Duprez E, Wagner K, Koch H, Tenen DG. C/EBPbeta: a major PML-RARA-responsive gene in retinoic acid-induced differentiation of APL cells. EMBO J, 2003, 22: 5806-5816

4 Jones L, Wei G, Sevcikova S, Phan V, Jain S, Shieh A, Wong JC, Li M, Dubansky J, Maunakea ML, Ochoa R, Zhu G, Tennant TR,
Shannon KM, Lowe SW, Le Beau MM, Kogan SC. Gain of myc underlies recurrent trisomy of the myc chromosome in acute promyelocytic leukemia. J Exp Med, 2010, 207: 2581-2594

5 Zeng CW, Chen ZH, Zhang XJ, Han BW, Lin KY, Li XJ, Wei PP, Zhang H, Li Y, Chen YQ. MIR125B1 represses the degradation of the PML-RARA oncoprotein by an autophagy-lysosomal pathway in acute promyelocytic leukemia. Autophagy, 2014, 10: 1726-1737

6 Zeng $\mathrm{C}, \mathrm{Xu} \mathrm{Y}, \mathrm{Xu} \mathrm{L}, \mathrm{Yu} \mathrm{X}$, Cheng J, Yang L, Chen S, Li Y. Inhibition of long non-coding RNA neat1 impairs myeloid differentiation in acute promyelocytic leukemia cells. BMC Cancer, 2014, 14: 693

7 Zeng CW, Zhang XJ, Lin KY, Ye H, Feng SY, Zhang H, Chen YQ. Camptothecin induces apoptosis in cancer cells via microRNA-125b-mediated mitochondrial pathways. Mol Pharmacol, 2012, 81: 578-586

8 Skvarova Kramarzova K, Fiser K, Mejstrikova E, Rejlova K, Zaliova M, Fornerod M, Drabkin HA, van den Heuvel-Eibrink MM, Stary J, Trka J, Starkova J. Homeobox gene expression in acute myeloid leukemia is linked to typical underlying molecular aberrations. $\mathrm{J}$ Hematol Oncol, 2014, 7: 94

9 Chen S, Liu S, Xu L, Yang L, Jin Z, Ma Y, Li B, Wu X, Yang J, Ma Y, Li Y. The characteristic expression pattern of BMI-1 and SALL4 genes in placenta tissue and cord blood. Stem Cell Res Therapy, 2013, 4: 49

10 Wang Q, Cheng T. Evidences for mutations in the histone modifying gene SETD2 as critical drivers in leukemia development. Sci China Life Sci, 2014, 57: 944-946

11 Zeng C, Yu X, Lai J, Yang L, Chen S, Li Y. Overexpression of the long non-coding rna pvt1 is correlated with leukemic cell proliferation in acute promyelocytic leukemia. J Hematol Oncol, 2015, 8: 126

12 Lehtonen A, Ahlfors H, Veckman V, Miettinen M, Lahesmaa R, Julkunen I. Gene expression profiling during differentiation of human monocytes to macrophages or dendritic cells. J Leukoc Biol, 2007, 82: $710-720$

13 Costa V, Angelini C, De Feis I, Ciccodicola A. Uncovering the complexity of transcriptomes with RNA-seq. J Biomed Biotechnol, 2010, 2010: 853916

14 Mao Y, Xiong L, Wang S, Zhong J, Zhou R, Li L. Comparison of the transcriptomes of mouse skin derived precursors (SKPs) and skp-derived fibroblasts (SFBs) by RNA-seq. PLoS One, 2015, 10: e0117739

15 Lan D, Xiong X, Wei Y, Xu T, Zhong J, Zhi X, Wang Y, Li J. RNA-seq analysis of yak ovary: improving yak gene structure information and mining reproduction-related genes. Sci China Life Sci, 2014, 57: 925-935

16 Ren S, Peng Z, Mao JH, Yu Y, Yin C, Gao X, Cui Z, Zhang J, Yi K, $\mathrm{Xu} \mathrm{W}$, Chen C, Wang F, Guo X, Lu J, Yang J, Wei M, Tian Z, Guan Y, Tang L, Xu C, Wang L, Tian W, Wang J, Yang H, Sun Y. RNA-seq analysis of prostate cancer in the chinese population identifies recurrent gene fusions, cancer-associated long noncoding RNAs and aberrant alternative splicings. Cell Res, 22: 806-821

17 Chen S, Yang P, Jiang F, Wei Y, Ma Z, Kang L. De novo analysis of transcriptome dynamics in the migratory locust during the development of phase traits. PLoS One, 5: e15633

18 Daigneault M, Preston JA, Marriott HM, Whyte MK, Dockrell DH. The identification of markers of macrophage differentiation in PMA-stimulated THP-1 cells and monocyte-derived macrophages. PLoS One, 2010, 5: e8668

19 Busca A, Saxena M, Iqbal S, Angel J, Kumar A. PI3K/Akt regulates survival during differentiation of human macrophages by maintaining NF-kappaB-dependent expression of antiapoptotic Bcl-xL. J Leukoc Biol, 2014, 96: 1011-1022

20 Debbas V, Arai RJ, Ferderbar S, Schindler F, Stern A, Monteiro HP. Regulation of p21Waf1 expression and TNFalpha biosynthesis by glutathione modulators in PMA induced-THP1 differentiation: involvement of JNK and ERK pathways. Biochem Biophys Res Commun, 2007, 363: 965-970

21 Dennis G Jr., Sherman BT, Hosack DA, Yang J, Gao W, Lane HC, 
Lempicki RA. David: database for annotation, visualization, and integrated discovery. Genome Biol, 2003, 4: P3

22 Theilgaard-Monch K, Jacobsen LC, Borup R, Rasmussen T, Bjerregaard MD, Nielsen FC, Cowland JB, Borregaard N. The transcriptional program of terminal granulocytic differentiation. Blood, 2005, 105: 1785-1796

23 Luo XH, Guo LJ, Yuan LQ, Xie H, Zhou HD, Wu XP, Liao EY. Adiponectin stimulates human osteoblasts proliferation and differentiation via the MAPK signaling pathway. Exp Cell Res, 2005, 309: 99-109

24 Sriuranpong V, Borges MW, Ravi RK, Arnold DR, Nelkin BD,
Baylin SB, Ball DW. Notch signaling induces cell cycle arrest in small cell lung cancer cells. Cancer Res, 2001, 61: 3200-3205

25 Traore K, Trush MA, George M Jr., Spannhake EW, Anderson W, Asseffa A. Signal transduction of phorbol 12-myristate 13-acetate (PMA)-induced growth inhibition of human monocytic leukemia THP-1 cells is reactive oxygen dependent. Leuk Res, 2005, 29: 863-879

26 Pulukuri SM, Rao JS. Activation of p53/p21Waf1/Cip1 pathway by 5-aza-2'-deoxycytidine inhibits cell proliferation, induces pro-apoptotic genes and mitogen-activated protein kinases in human prostate cancer cells. Int J Oncol, 2005, 26: 863-871

Open Access This article is distributed under the terms of the Creative Commons Attribution License which permits any use, distribution, and reproduction in any medium, provided the original author(s) and source are credited.

\section{Supporting Information}

Figure S1 PMA induces cell differentiation in THP1. Cell morphology was observed under a phase-contrast microscope and photographed by a digital camera.

Table S1 Real-time PCR primers

Table S2 Gene expression level in THP1 cells with or without PMA treatment

Table S3 GO-Biological Process analysis of DEGs in THP1 cells with or without PMA treatment

Table S4 KEGG pathway analysis of DEGs in THP1 cells with or without PMA treatment

Table S5 Gene expression level of BCL2 family in THP1 cells with or without PMA treatment

The supporting information is available online at life.scichina.com and link.springer.com. The supporting materials are published as submitted, without typesetting or editing. The responsibility for scientific accuracy and content remains entirely with the authors. 